\title{
As the Medium and Sub-culture: Rethink the MOOC’s Explorations in the Liberal Arts in a University From the Perspectives of Social Epistemology*
}

\begin{abstract}
KAN Wei
Beijing Normal University, Beijing, China

In this paper, the researcher examines lessons from massive open online courses (MOOCs) in the liberal art in a university. It intends to define a research agenda into the social epistemology impact of MOOCs, in order to reflect on changes in academic sub-culture and on learning measurement among students in university. For this reason the researcher suggests focusing attention on three features of MOOC phenomenology: MOOCs as a social epistemology —an active policy initiative to promote greater democratization of education; MOOCs as a medium and an academic sub-cultural artifact (mediated texts, videos, interface, platform functionalities) able to convey learning to distant learners; and, lastly, MOOCs as a measurement-in other words as instrumentation (i.e., learning statements, analytics, algorithms, visualizion tools, dashboards etc.) that allows students to monitor, analyze, and optimize the effectiveness of online teaching and learning. The paper also highlights their limits in these regards based on the perspectives of social epistemology.
\end{abstract}

Keywords: MOOC, social epistemology, academic sub-culture

\section{Background of Study}

With the rapid development of network technology, it is inevitable that educational resources will be online and open. In 2008, Dave Cormier and Professor Bryan Alexander first proposed the concept of massive open online course (MOOC) (McAuley, Stewart, Siemens, \& Cormier, 2010). In 2012, Stanford University, MIT, Harvard University, and other famous universities have used the Coursera, Udacity, and edX platforms to provide high-quality, free, and open online education resources, attracting many learners from all over the world to participate in online courses. Laura Pappano, author of The New York Times, called 2012 "the first year of MOOC” (Zhang \& Chen, 2018). The emergence of MOOC has attracted the attention of countries all over the world and well-known universities. For example, France launched the national digital platform FUN-MOOC in 2013 and implemented 18 digital initiatives, which promoted the development of French higher education MOOC (Zeng \& Zhang, 2018); The University of Melbourne has opened 22 MOOCs on the Coursera platform. Since the rise of China's MOOC in 2013, China's MOOC construction has made great progress, including Tsinghua University’s “School Online”, Shanghai Jiaotong University’s “Good University

\footnotetext{
* This research is supported by the funds of humanity and social research of Ministry of Education; Project Number is 14 YJA880027.

KAN Wei, Ph.D., Associated Professor, Faculty of Education, Beijing Normal University, Beijing, China.
} 
Online”, Chinese University MOOC. And the domestic basic education MOOC platform such as Ai Xuetang and C20 Mutual Union, each platform has its own characteristics, attracting many famous schools in China, Taiwan and Hong Kong to enter the MOOC platform, covering different courses of pre-service and post-employment, such as science, engineering, agriculture, medicine, education, psychology, etc.; wonderful content, high quality micro video, and interactive teaching have attracted a large number of learners to participate in learning, and have received good teaching benefits. At the same time, many scholars have carried out research on the connotation and classification of MOOCs, teaching methods, and learning evaluation systems, and draw useful conclusions (Wang \& Zhang, 2018).

After years of discussion around MOOC pedagogies and paradigms, the concept is about to change again. The massiveness and openness ideas are loosing their centrality in the public debate, while pressure for sustainability and for quality of learning design and pedagogy increases. In 2012, with the development of bespoke MOOC platforms, the phenomenon gained momentum (Daniel, 2012; McKenna, 2012) and reached new heights (Dillenbourg, 2013; Horn \& Christensen, 2013). In Europe, expectations were accompanied by a certain apprehension regarding the future of public universities, and competition among old and new players (Fundación Telefónica, 2015).

In fact, the MOOC in universities or colleges had been experiencing dramatic growth. A report from Class Central (Dhawal, 2015; 2016) showed that not only is the number of MOOCs increasing in university system, but so the number of students who enroll in open courses. In the year 2015, there was a global offer of over 4,000 courses; in 2016 the global offer was about 6,850 MOOCs from over 700 universities. The number of students has doubled from 16-18 million to 35 million in 2015. In 2016, the number of students enrolled in at least one MOOC reached 58 million, and 23 million students enrolled for the first time in a MOOC. However, several surveys of MOOC users agree that the most typical course participant is a male with a bachelor's degree who is 26 or older. In a survey of 400,000 users in 2015 by the University of Pennsylvania, the number of MOOC users with a degree reached 83\%; most are employed and living in industrialized countries (Gaebel, Kupriyanova, Morais, \& Colucci, 2018). These data clearly demonstrate that there is a high demand for education that is not being met by the education system as it stands. Moreover, they also demonstrate that already privileged people are taking advantage of this free educational offer, thus opening the door to criticism about the capacity of online education to reduce inequalities and to provide access to education for all. The United States are still leading the trend, with Coursera and edX providing over $50 \%$ of the global offer, while in Europe providers such as Future Learn and FUN are leading the European MOOC offer; a further contribution in fostering MOOC development is coming from new regional platforms. The Arabic-language platform, Edraak, backed by the Queen Rania Foundation of Jordan, is approaching one million users; India launched its official MOOC platform, Swayam, with six million users, while the Chinese Xuetang Zaixian is the only non-English MOOC platform in the top three, reaching 10 million users in 2018 (Marsh, 2019).

In Europe, survey studies have highlighted a complex situation, with some areas much more active than others but also a wide range of key players (public, private, non-profit), some of whom have different learning objectives and interactive models (De Rosa, 2017). A comprehensive study of the European scenario has been conducted by the European Universities Association to gain a better understanding of the strategic reasons why a higher education institution is or is not involved in MOOCs (Gaebel, Kupriyanova, Morais, \& Colucci, 2018), and to compare these reasons with the results of similar studies in United States (Allen \& Seaman, 2014; 2015). 
The survey demonstrated that more institutions in Europe than in the United States plan to increase their MOOC offer in the near future. Opportunities for pedagogical experimentation and branding are primary objectives for the European Union, whereas objectives related to finance or scalability are of less importance. On the opposite side, United States institutions aim to boost student recruitment.

In the modern context, the liberal arts education reforms had been processed since 2010 in Chinese universities or colleges. The typical liberal arts subjects are on the broad scope of the category including the humanities, as well as social, natural, and formal sciences. There are differences in the particular subjects included in liberal arts degree programs at different institutions. However, the liberal arts spectrum is generally accepted as literature, linguistics, philosophy, history, psychology, law, sociology, economics, geography, etc.

With this tendency towards growth and increasing interest, the delivery mode and access to education is definitely going to shape and change the educational landscape at many levels. Porter (2015) believes that we cannot be sure about the quality or the quantity of disruption that MOOCs are going to bring, but educational institutions need to experiment with them and to assess the impact on their internal operation.

\section{Purpose of Research}

\section{Research Questions}

In line with the argument about the mismatch between a social epistemology construct and an open MOOC context, the present study was designed to address two research questions:

RQ1: What does students' learning behaviors in MOOCs look like when evaluated across the entire group of forum users?

RQ2: What is the association between student perceptions of social epistemology and their levels of participation in MOOC discussion forums, and does this perception differ across the groups of learners with varying participation patterns?

By addressing these questions, this study demonstrates whether or not different ways of evaluating social epistemology bring consistent insights. Answering the second research question will help establish the relationship between student's self-reported perceptions of social epistemology and the log data derived from their posting activity.

\section{Research Method and Ethical Consideration}

Student perceptions of social epistemology were collected through the questionnaire (Arbaugh \& Benbunan-Fich, 2006). To better understand the association between levels of participation in MOOC forums and student perceptions of social epistemology, the researcher compared self-reported levels of social epistemology between the groups with varying levels of forum activity: forum participants who consistently posted on the forums, those who posted occasionally, and those who did not post. The design of the study was exploratory, as the researcher wanted to understand if the reported social epistemology would differ across these three groups, as well as if it would differ from the averages reported on the entire sample of respondents.

The survey was implemented in three MOOCs system run by the one of normal universities in Beijing in 2017: (1) Basic Principles of Education (BPE); (2) Introduction to Traditional Culture (ITC); and (3) Creative Writing (CW). The MOOCs differed in subject, duration, and pedagogical design in relation to forum activity. To account for course instructional, disciplinary, and contextual specificity, the analysis was done for each course separately. All students were invited to participate in a post-course survey administered in the final 
weeks of each course. In all three courses, no specially designed learning interventions were incorporated to facilitate interpersonal interactions.

"Ethical issues" is the last key area emerging from the public debate and includes the academic duties of care and integrity. Using the words of Marshall (2014), ethics in the MOOC environment is at least twofold, deontological, and teleological. The collection of massive data regarding MOOC users in universities or colleges which is basically unregulated could prove to be a significant sector for further digging in the data, and could threaten individual and privacy rights. Existing privacy protection mechanisms include anonymization of data and consent (in this case it makes data collection hard), which may not be enough, especially when it's not completely clear or well defined who the data belong to or could be used by the educational research. On the teleological level, as Marshall insisted, "we have a professional and social obligation to ensure that we are not abusing a position of trust and responsibility and acting, irrespectively of our wider goals and intentions, in an unethical manner” (Marshall, 2014, p. 2). Taking this background into consideration, this study intends to define a research agenda on the impact of the MOOC movement academic culture, and on learning assessment. A key part of the agenda recognizes the relevance that social epistemology plays in advancing educational reform. As the research questions proposed in this study highlight, MOOCs constitute a unique and somewhat independent force having significant implications for society and how we think about higher learning.

\section{Data Description}

To account for the varying frequency and quality of participant forum use, survey respondents were divided into three groups based on the regularity of their forum activity rather than the volume of such activity. Poquet (2017) demonstrated that learners who participate regularly co-occur with one another over the course duration - that is, individuals posting with certain regularity are also defined by the continuity of interactions, much like cohorts inside formal online courses.

The first group, regular posters, comprised all MOOC learners making forum posts for three weeks or more (Table 1). If, for example, a learner made one post in Week 1, three posts in Week 5, and 20 posts in Week 10, this person would be considered a regular poster. The division into regular and occasional posters was based both on data exploration and latent class analysis.

Table 1

Data Description: Summary of the Sample

\begin{tabular}{|c|c|c|c|c|c|c|c|c|}
\hline Course & $\begin{array}{l}\text { Enrolled } \\
\text { students }\end{array}$ & $\begin{array}{l}\text { Course } \\
\text { duration } \\
\text { (weeks) }\end{array}$ & $\begin{array}{l}\text { Regular } \\
\text { forum } \\
\text { posters }\end{array}$ & $\begin{array}{l}\text { Occasional } \\
\text { forum } \\
\text { posters }\end{array}$ & $\begin{array}{l}\text { Forum } \\
\text { contributors } \\
\text { (total) }\end{array}$ & $\begin{array}{l}\text { Survey } \\
\text { responses } \\
\text { (total) }\end{array}$ & $\begin{array}{l}\text { Regularly- } \\
\text { posting } \\
\text { respondents }\end{array}$ & $\begin{array}{l}\text { Occasionally- } \\
\text { posting } \\
\text { respondents }\end{array}$ \\
\hline BPE & 279 & 18 & 14 & 54 & 68 & 213 & 18 & 29 \\
\hline ICT & 312 & 18 & 18 & 69 & 87 & 277 & 117 & 243 \\
\hline CW & 154 & 18 & 12 & 32 & 44 & 111 & 27 & 137 \\
\hline
\end{tabular}

Course 1: Basic Principle of Education (BPE) of MOOC was delivered by the Faculty for Education in this university. The 18-week course had 279 registered participants, with 263 course certificates awarded. The course employed interactive tasks, and learners were encouraged to discuss these on the forum. Peer assessment was a part of the course design. Student forum activity in the course decreased from 93 contributions in the first week to 67 in Week 4 (see Table 2, below). The course activity of BPE decreased in volume, with a small group of 14 regular posters repeatedly contributing to the forum for three or more weeks of the course (see 
Table 1, above). At the end of the course, a total of 213 respondents completed the questionnaire, with 18 of them being regular contributors, 29 occasional contributors, and 37 non-posters. From those who completed the questionnaire, more than $70 \%$ of MOOC participants obtained the certificates; the remaining respondents also included learners who started the course but did not complete it, and those who audited parts of the course.

Course 2: Introduction to Traditional Culture (ITC) was delivered by the History Department of the university. The 18-week course was based on the discussion on the issues of traditional culture. The course assessed learner knowledge through discussion for the forum activity, except that the course instructor and several teacher assistants actively communicated with the students on the forum. The course enrolled 312 students and certified 277 of them. The volume of forum activity decreased from Week 2 to Week 18, but the decrease was not as drastic as that observed in BPE. The volume of interactions fluctuated between 10 and 40 interactions weekly, produced by some 20 to 40 students (see Table 2, below). Thirteen participants interacted with others every week of the course, and the group of regular contributors comprised 177 participants (see Table 1, above).

Table 2

The Number of Posts and Posters in Week of Dual of the Course

\begin{tabular}{|c|c|c|c|c|c|c|c|c|c|}
\hline Course & Week 2 & Week 4 & Week 6 & Week 8 & Week 10 & Week 12 & Week 14 & Week 16 & Week 18 \\
\hline \multicolumn{10}{|c|}{ Number of forum contributions per week } \\
\hline BPE & 93 & 67 & 41 & 25 & 18 & 11 & 9 & 7 & 11 \\
\hline ITC & 71 & 52 & 33 & 21 & 12 & 9 & 6 & 14 & 11 \\
\hline CW & 39 & 25 & 35 & 12 & 8 & 6 & -- & -- & -- \\
\hline \multicolumn{10}{|c|}{ Number of posters contributing per week } \\
\hline BPE & 79 & 64 & 67 & 31 & 28 & 24 & 13 & 58 & 16 \\
\hline ITC & 34 & 22 & 21 & 18 & 11 & 7 & 12 & 15 & -- \\
\hline CW & 53 & 64 & 46 & 38 & 30 & -- & -- & -- & -- \\
\hline
\end{tabular}

Questionnaires which had been conducted at the end of the course were completed by 745 individuals, 44 of whom were regular contributors, 155 occasional contributors, and 546 non-posters. The respondents mostly represented the "silent Chinese students" (Helena, 2011) completers, but also included people auditing the course and students who disengaged at some point during the course.

Course 3: Creative Writing (CW). This 18-week course was delivered by the Department of Chinese Literature. It enrolled 154 students. Of the total enrolled student cohort 149 received certificates of completion. CW was as the same time in duration with BPE and ITC. The course was designed so that each week had a distinct focus, with the course outcomes being assessed through weekly writing section. More discussion and displaying activities were designed to facilitate forum participation. Forum activity in the CW course experienced a steep drop in activity not only from the second to the fourth week of the course, but the interaction nearly stopped at the last six weeks (see Table 2, above). No MOOC participants in this course interacted with another participant. That is, the group of regular posters comprised a very small percentage of all forum users, and the volume of activity in this course was smaller than in the first two courses.

A series of nonparametric Kruskal-Wallis tests for each of the nine survey items investigated whether there were significant differences in social epistemology model scores among the groups of students. Given that the questionnaire items used an ordinal Likert scale, and that there were differences in the size of the three groups of students, we opted for the use of nonparametric significance tests. To control for the inflated Type I 
error rate that may result from multiple consequent comparisons, we used the Holm-Bonferroni correction procedure (Holm, 1979). This procedure offered more statistical power than the ordinary Bonferroni correction while preserving a strict control over family-wise error rate (FWER) (Holm, 1979). The significant Kruskal-Wallis tests $(\mathrm{p}<0.05)$ were then followed up with a Dunn test, also with a Holm-Bonferroni correction.

\section{Data Analysis}

The results of questionnaire suggest that MOOC learners who completed the post-course questionnaire established some degree of social epistemology model. The average scores for different survey questions varied between 2.7 to 3.9 (on a 1-5 scale). Unfortunately, these scores cannot be statistically compared to those previously reported in the studies of formal online education (since the scores represent ranked data within the subgroups of varying size). From a descriptive point of view, social epistemology model scores previously reported in formal online courses were typically higher than 3.0 and are collected from groups of 10 to 15 people interacting in courses lasting for up to 16 weeks. For instance, Akyol and Garrison (2008) reported a range of 3.94 to 4.30; the students in the study by Swan et al. (2008) yielded a mean social epistemology model score of 4.18. Other studies have observed ranges of 3.67 to 4.06 (Maddrell, Morrison, \& Watson, 2011). It appears that in the examined MOOC settings, where larger groups of learners interacted for a shorter time than in previously-examined formal online courses, social epistemology model could be established. This generally supports the observations of previous work investigating social epistemology model in MOOCs.

Consistent patterns of social epistemology model evaluation are observed across the courses, with affective expression (Q1-Q3) being rated as lowest and open communication (Q4-Q6) rated as highest. In their analyses of social epistemology model development over time, Akyol and Garrison (2008) pointed out that open communication, along with affective expression, is the most prominent in the initial stages of social epistemology model development, whereas group cohesion slowly grows over time as the group identity forms. It is, then, noteworthy that the affective expression of the learners yielded the lowest scores, which is in contrast to what would be expected from formal online settings. Reported open communication and group cohesion, however, can be interpreted as aligned with what has been observed in other educational contexts.

There are also substantial differences in the levels of social epistemology model established. The size of the posting cohort and the duration of the MOOC seem to be associated with the levels of social epistemology model reported across the entire sample. The highest scores for social epistemology model (range: 3.2-3.7) were reported by the participants in the analyzed MOOCs with the smallest number of total participants (i.e., $\mathrm{CW})$. Besides having the smallest group of regular posters, ITC had a sizable volume of forum interactions. In contrast, the size of the regular cohort and volume of interactions in TPM course was similar, but the range of average social epistemology model was slightly lower (2.9-3.5).

The properties of analyzed MOOCs (i.e., size of the cohort, duration of the course, and the volume of interactions) may explain the differences among the social epistemology model scores, irrespective of consideration for pedagogies that contextualize interpersonal interactions. The importance of the course duration was similarly highlighted in the meta-analysis by Richardson, Maeda, Lv, and Caskurlu (2017) who found that the duration of the course mediated between the social epistemology model and satisfaction. In the analyzed courses, the instructors have not given specific tasks that integrated the use of forums, and forum participation was not graded. Although CW had embedded peer assessment within the course, our look at the 
discussions on the forum suggested that the discipline of the course had more to do with its heightened interactivity.

The most important observation to be drawn is that the levels of social epistemology model reported from the entire sample do not offer an accurate representation of the levels of social epistemology model established in the course. The average social epistemology model scores are skewed by the lower levels reported by the non-posting students, and by the higher levels reported by the regular forum posters. Had we only examined average levels of social epistemology model for the whole of each course, as has been done in the previous research of social epistemology model in MOOCs, BPE and ITC posters would appear to have established higher levels of social epistemology model, while the largest cohort in the CW course lagged behind. However, such observation is inaccurate since the social epistemology model construct (conceptually) examines perceptions of those who can establish social epistemology model in principle-that is, those who interact regularly.

A comparison of the social epistemology model scores reported by the regular posters suggests that the BPE regular posters' scores are more aligned with the levels of social epistemology model in the ITC course, rather than those of CW. That is, regular posters in BPE developed lower perceptions of open communication and group cohesion than regularly-participating students undertaking CW-and this despite a much larger cohort size in CW. Given that ITC had a smaller cohort posting, this should indicate that the social interactions within the course could have been less vibrant than expected. In conclusion, looking at the social epistemology model scores by group demonstrates that CW and BPE were rather successful in terms of social epistemology model, whereas TPM was less successful than expected.

As discussed, a valid comparison of social epistemology model raw scores among the learner groups of varying engagement levels can be accomplished through the statistical analysis of differences between the groups. If applied to the entire cohort, social epistemology model scores do not offer an accurate representation of perceptions from the regularly-participating learners.

\section{Discussion}

The patterns reported above were used as exploration. Further statistical comparison of the effect of participation levels on the social epistemology model scores of each course revealed that student engagement levels was associated with differences in the perception of social epistemology model. However, such a conclusion was not consistent across all analyzed cases.

This study also intends to involve scholars from educational research community in a debate that has been largely ignored, since the topic of the changing educational environment as a result of MOOCs has been largely considered in terms of delivery models or learning patterns, or at best, learner demographics, and so has interested pedagogists and educational sociologists. It could also be useful to analyze the MOOC phenomenon by focusing on emerging policy frameworks and changing power relationships between old and new actors. While, on the other hand, media scholars could use their lens to look at mediated educational processes, exploring whether the inherent logic of action is affected by the different media used. The objective is to assess the transformative power of MOOCs in education.

Where all of the above are concerned, as well as the other cases that will probably emerge over time, it is important to understand how these players will face up to the new opportunities and threats posed by the Open Education movement as it goes global and in what way they intend to respond to that evolution. At this stage, 
two models seem to be emerging. One is the schooling as platform approach, which identifies online teaching as an extra effort —and an opportunity - to reflect on the changing world of learners and learning. This world seems widely affected by the so-called uberization of teaching, a concept used to identify what happens when a specific student—autonomously—seeks out a specific teacher or group of teachers to learn a specialized skill or skill set (Rogers, 2014) and, vice versa, when a teacher or a specific group of teachers offer their knowledge on the market, outside institutional or formalized arrangements.

On the opposite side, the platform as brand extension approach intends to position educational institutions on the global market of education by exploiting the channel capability of a few relevant MOOC aggregators to reach new audiences and by accepting unification of their pedagogical approaches in a pre-established format. Both models represent a challenge for which outcomes are uncertain.

This research implies the analysis of the role of private as well as public key players/stakeholders in the field and/or in relation to the MOOC movement at a European, international as well as national level (who); the policy framework, values, and paradigms which they are inspired by (why); the strategies, policies, and goals they enact to react to/sustain the change that the virtualization of teaching implies (how); the changing habits of education targets in the digital era and their demand for highly personalized learning paths and new rights (to whom); the impact that MOOCs are expected to have on organizational settings and academic culture in general, on institutional performances, and market assets (impact); the effects in terms, for example, of legitimation processes (for new agency, actors, pedagogies etc.) and the side-effects in terms of medialization vs. disintermedialization of education from their traditional agencies, i.e., the urbanization of education, the starization of teachers (effects).

\section{Conclusion}

Due to the profound implications that educational change is going to have on 21st century learning, the definition of new policy settings that are able to anticipate, organize, and realign educational needs with individual rights is becoming urgent (De Rosa, 2017). Research can play a fundamental role by addressing and informing policymakers in a sensitive field that can affect the future of generations of people as well as their quality of life in a fast changing society. In this respect, questioning the MOOC phenomenon according only to pedagogies and learning theories means underestimating it. This is the reason why we suggest focusing attention on three features of MOOC phenomenology as it manifests itself: the MOOC as social movement, as medium, and as measurement.

MOOC as movement is a social epistemology towards clear directions: with online learning, the idea of tomorrow's education, where any educational need is satisfied at a distance for all, in real time, and on demand is already there. But the time has arrived to reflect on the road that the MOOC experience has taken us down so far: For example, what will be the destiny of education in the artificial intelligence era, when AI applications supported by big data and analytics could decide the type and quality of investment deserved by each individual? The MOOC experience in this university demonstrated that teachers can be replaced by good actors able to talk on video in a more appealing way. In the future, are we really sure that teaching will not be replaced by robots?

MOOC as medium implies a transformative power of technology over culture [and vice versa?], where transactional pedagogies and system unbundling will contribute to redefine roles, functions, and power relationships between old and new actors, raising lots of ethical issues. 
Finally, MOOC as measurements is a decisive step towards quantified education, as is expressed by learning, institutional and academics analytics through their "ranking of everything” procedures. Here the power of algorithms, the quality of teaching, and learner performances may represent a unique entry point for a new and sneakier system of inequalities. Because, to some extent, the MOOC phenomenon redefines what is meant by "learning”, "teaching”, and "assessment" and at the same time blurs the boundaries between them.

By widening the disciplinary horizons around the MOOC phenomenon, this research agenda offers the opportunity to identify emerging issues at the macro, meso, and micro level. At the macro level, by observing how the phenomenon evolves and what is the impact exercised by both the market and public institutions, the research agenda could estimate the real disruptive potential of the MOOC phenomenon, highlighting those turning points in which a strategy stops being disruptive and becomes only aggressive. At the meso level, understanding how MOOC initiatives intend to cope with cultural diversity and how the pressure to scale will be harmonized with different pedagogies, languages, and personalization requests, the agenda will help to address implications at a deeper level where identities and differences are concerned. Finally, at the micro level, by conceiving privacy and civil rights as a politically sensitive territory, this research agenda could represent an enforcement of the rule of law where evidence based analysis is concerned.

\section{References}

Akyol, Z., \& Garrison, D. R. (2008). The development of a community of inquiry over time in an online course: Understanding the progression and integration of social, cognitive and teaching presence. Journal of Asynchronous Learning Networks, 12(3-4), 3-22.

Allen, I. E., \& Seaman, J. (2015). Grade change: Tracking online education in the United States. Babson Survey Research Group and Quahog Research Group. Retrieved from onlinelearningsurvey.com.

Arbaugh, J. B., \& Benbunan-Fich, R. (2006). An investigation of epistemological and social dimensions of teaching in online learning environments. Academy of Management Learning \& Education, 5(4), 435-447.

Daniel, J. (2012). Making sense of MOOCs: Musings in a maze of myth, paradox and possibility. Retrieved from https://linc.mit.edu/linc2013/proceedings/Plenary-Presentations/Daniel.pdf

De Rosa, R. (2017). Governing by data: Some considerations on the role of analytics in education. In C. Lauro, E. Amaturo, M. G. Grassia, B. Aragona, and M. Marino (Eds.), Data science and social research-epistemology, methods, technology and applications (pp. 67-77). Cham, CH: Springer International.

Dhawal, S. (2015). By the numbers, MOOCs in 2015. Class Central Report 2015. Retrieved from https://www.class-central.com/

Dhawal, S. (2016). By the numbers, MOOCs in 2016. Class Central Report 2016. Retrieved from https://www.class-central.com/

Dillenbourg, P. (2013). MOOCs in Europe: An overview. Presentation at EMOOCS European Stakeholder Summit, Lausanne, 2013.

Fundación Telefónica. (3 February 2015). MOOCs in the education of the future: Digitizing training.

Gaebel, M., Kupriyanova, V. A., Morais, R., \& Colucci, E. H. (2018). E-learning in European higher education institutions: Results of a mapping survey conducted in October-December 2018. Retrieved from http://www.eua.be/Libraries/publication/e-learning_survey

Helene, S. (2011). Characteristics of Chinese students' learning styles. The Eighth of International Conference of Language, Media and Culture (ICLMC).

Holm, S. (1979). A simple sequentially rejective multiple test procedure. Scandinavian Journal of Statistics, 6(2), 65-70.

Horn, M., \& Christensen, C. (20 February 2013). Beyond the buzz, where are MOOCs really going? Wired.com. Retrieved from https://www.wired.com/2013/02/beyond-the-mooc-buzz-where-are-they-going-really/

Maddrell, J. A., Morrison, G. R., \& Watson, G. S. (November 2011). Community of inquiry framework and learner achievement. Paper presented at The Annual Meeting of the Association of Educational Communication \& Technology, Jacksonville, FL.

Marsh, N. (January 4, 2019). MOOC users reach 58 million globally. The Pie News. Retrieved from https://thepienews.com/news/edu-tech/mooc-users-reach-58-million-globally

Marshall, S. (2014). Exploring the ethical implications of MOOCs. Distance Education, 35(2), 250-262. 
McAuley, A., Stewart, B., Siemens, G., \& Cormier, D. (2010). The MOOC model for digital practice. Retrieved from http://www.e-learnspace.org/Articles/MOOC_Final.pdf [2013-04-16]

McKenna, L. (May 11, 2012). The big idea that can revolutionize higher education: "MOOC”. The Atlantic. Retrieved from https://www.theatlantic.com/business/archive/2012/05/the-big-idea-that-can-revolutionize-higher-education-mooc/256926/

Poquet, O., Dowell, N., Brooks, C., \& Dawson, S. (2018). Are MOOC forums changing? In LAK '18 Proceedings of the 8th International Conference on Learning Analytics and Knowledge, 340-349.

Porter, S. (2015). To MOOC or not to MOOC: How can online learning help to build the future of higher education? Oxford, UK: Chandos Publishing.

Richardson, J. C., Maeda, Y., Lv, J., \& Caskurlu, S. (2017). Social presence in relation to students' satisfaction and learning in the online environment: A meta-analysis. Computers in Human Behavior, 71, 402.

Rogers, G. (2014). The Uberization of Education. Retrieved from https://www.linkedin.com/ pulse/20140603135511-20348008-the-uberization-of-education/

Swan, K., Shea, P., Richardson, J., Ice, P., Garrison, D. R., Cleveland-Innes, M., \& Arbaugh, J. B. (2008). Validating a measurement tool of presence in online communities of inquiry. E-Mentor, 2(24), 1-12.

Wang, Y. G., \& Zhang, Q. (2018). MOOC: Characteristics and learning mechanism. Educational Research (Jiaoyu Yanjiu), 416(9), 112-120, 133.

Zeng, X. J., \& Zhang, H. (2018). The national strategy for the development of MOOC in France: An analysis of France Université Numérique. International and Comparative Education (Guoji Bijiao Jiaoyu), 336(1), 78-87.

Zhang, D. C., \& Chen, J. X. (2018). Curriculum analysis based on Chinese University MOOC platform. Education Journal, 7(3), 68-74. 